\title{
Surface-Induced Dissociation Mass Spectra as a Tool for Distinguishing Different Structural Forms of Gas-Phase Multimeric Protein Complexes
}

\author{
Royston S. Quintyn, Mowei Zhou, Jing Yan, and Vicki H. Wysocki* \\ Department of Chemistry and Biochemistry, Ohio State University, \\ 484 West 12th Avenue, Columbus, Ohio 43210, United States
}

\section{Supplemental Information}

The extent of dissociation of the precursor decreases as it becomes more unfolded.

Another interesting feature observed in the SID dissociation patterns of the various unfolding intermediates formed by cone activation of CRP, TTR and Con A is the increasing fraction of undissociated precursor that accompanies increasing cone voltage (Figure S1). CRP shows the largest increase in undissociated precursor from cone $50 \mathrm{~V}$ to $200 \mathrm{~V}$, whereas Con A shows the smallest. This trend tracks well with our results described earlier for CRP, TTR and Con A, which show that CID-induced unfolding of CRP at elevated cone voltages results in the largest shifts in CCS $\left(6550 \AA^{2}\right.$ at cone $50 \mathrm{~V} \rightarrow 7150 \AA^{2}$ at $200 \mathrm{~V})$ and average charge state of monomers $(+4.1$ at cone $50 \mathrm{~V} \rightarrow+9.1$ at cone $200 \mathrm{~V})$. Hence, we speculate that the partially unfolded CRP pentamer observed at elevated cone voltages is more flexible, and thus may be able to dissipate internal energy more efficiently leading to an increase in the energy needed for dissociation to occur. This phenomenon remains to be further examined. 


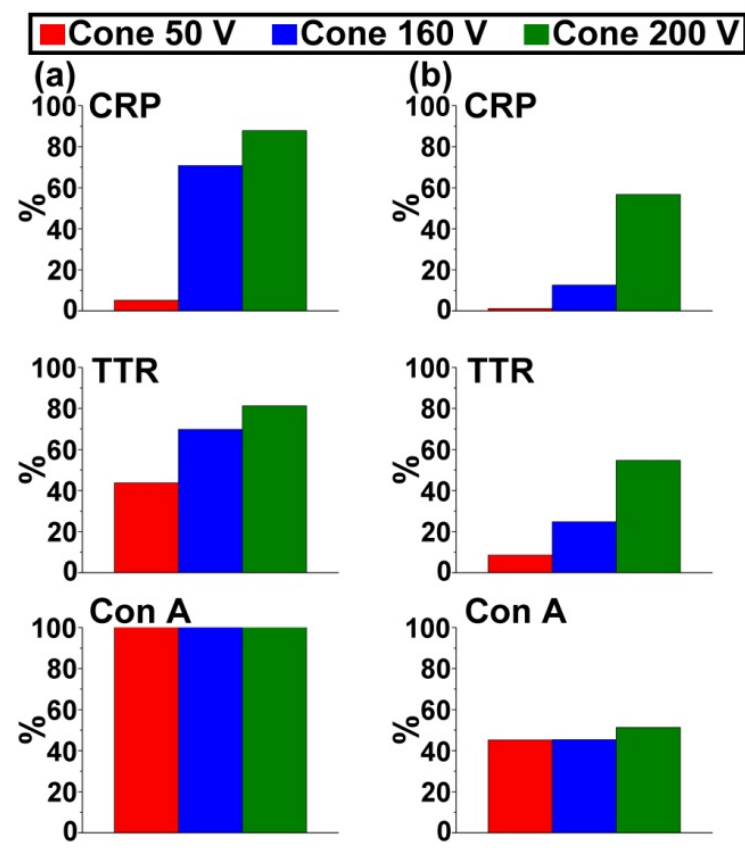

Figure S1 Effect of cone voltage on the fraction of undissociated precursor obtained from SID. Bar charts showing the change in fraction of undissociated precursor with cone voltage are shown for $+18 \mathrm{C}$ Reactive protein, +11 Transthyretin and +14 Concanavalin A at SID acceleration voltages of (a) $50 \mathrm{~V}$ and (b) $70 \mathrm{~V}$. 

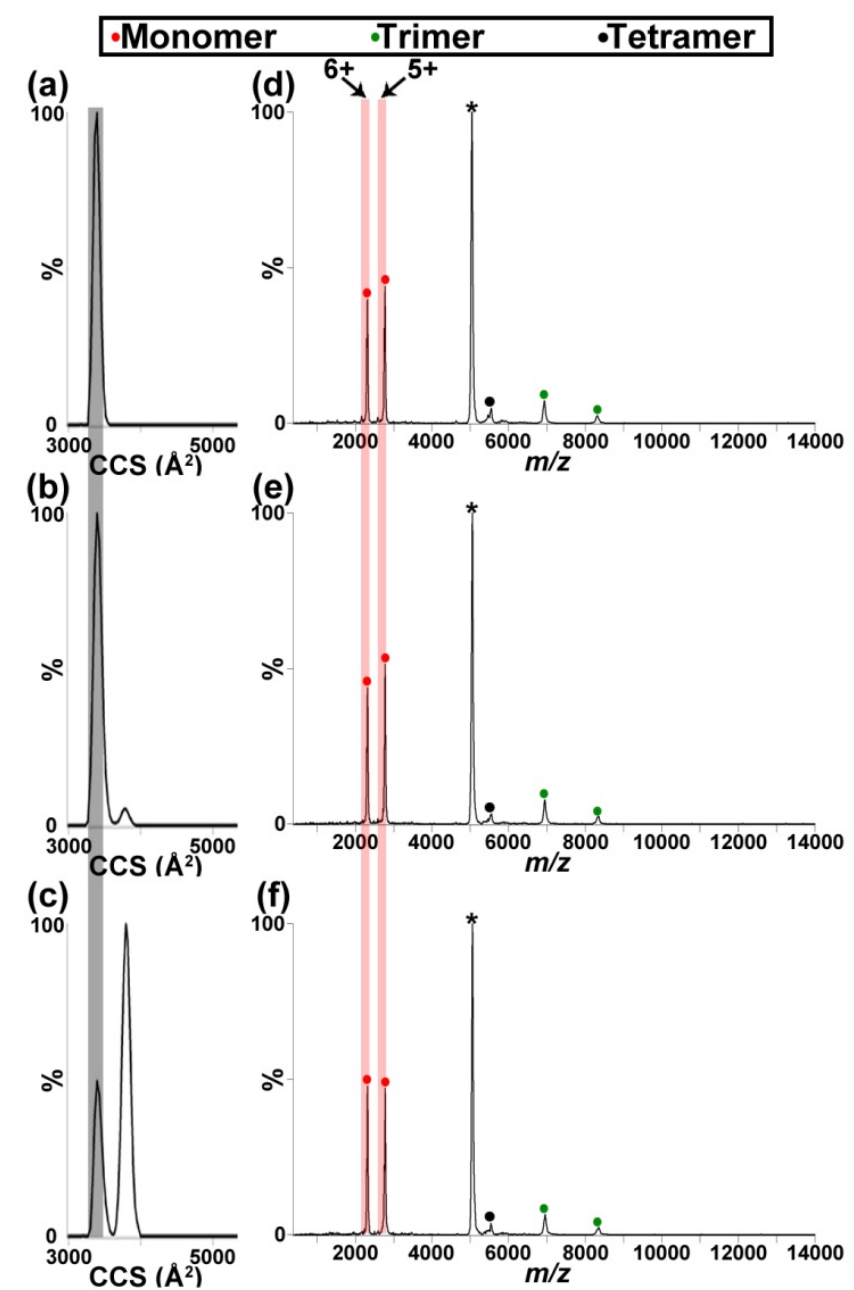

Figure S2: Effect of in-source collisional activation on the CID (1650 eV) dissociation products of TTR. CCS plots of the +11 TTR precursor at cone voltages of (a) $50 \mathrm{~V}$ (b) $160 \mathrm{~V}$ and (c) $200 \mathrm{~V}$. The theoretical CCS calculated using the TTR crystal structure is highlighted by the gray vertical line. Representative CID spectra are also shown at cone (d) 50V (e) $160 \mathrm{~V}$ and (f) 200V. CID yields monomers and complementary trimers regardless of cone voltage. The +5 and +6 monomer peaks are highlighted by the red vertical line.

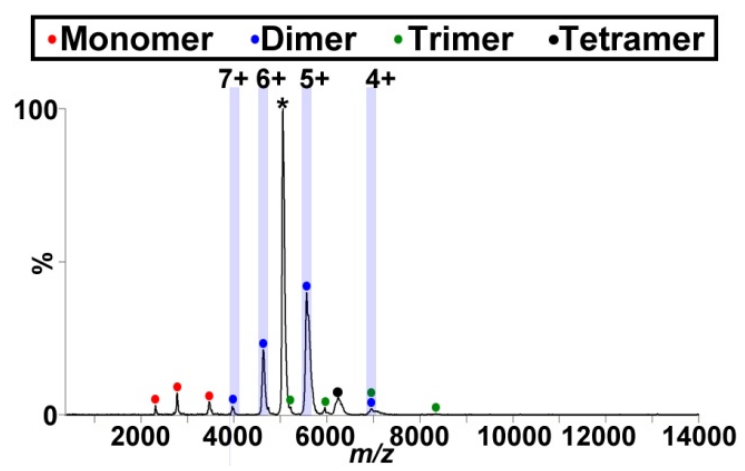

Figure S3: Low-energy SID of TTR yields primarily dimers. SID spectrum of the +11 TTR precursor at a collision energy of $550 \mathrm{eV}$ is shown. Peaks corresponding to the TTR dimers are highlighted in blue. 


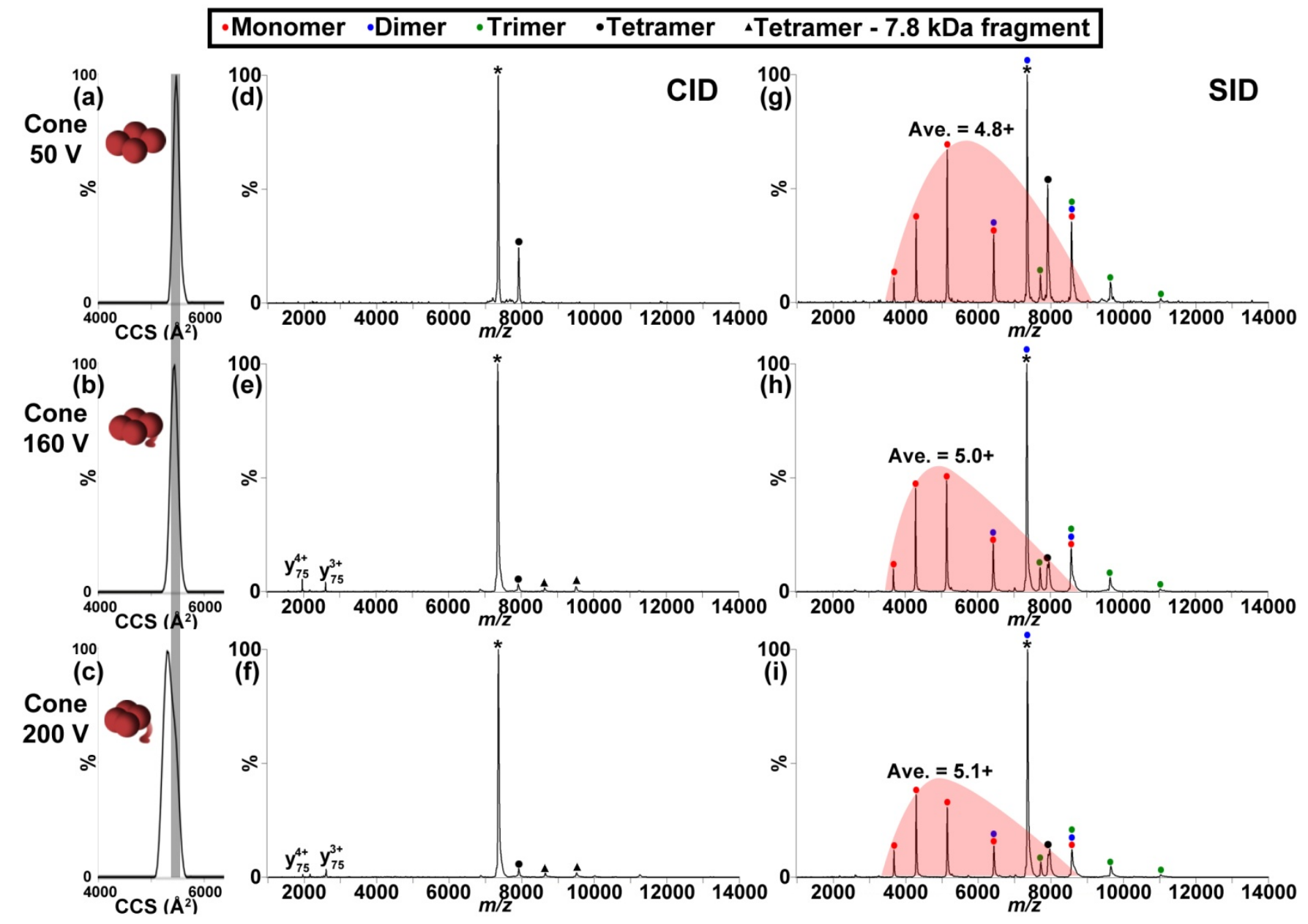

Figure S4: Effect of in-source collisional activation on the CID (1680 eV) and SID (1050 eV) dissociation products of Con A. CCS plots of the +14 Con A precursor at cone voltages of (a) $50 \mathrm{~V}$ (b) $160 \mathrm{~V}$ and (c) $200 \mathrm{~V}$. The theoretical CCS calculated using the Con A crystal structure is highlighted by the gray vertical line and cartoons representing the proposed structures of the various conformations present are shown in the inset. Representative CID and SID spectra are also shown for cone (d, g) 50V (e, h) 160V and (f, i) $200 \mathrm{~V}$ respectively. The average charge states are calculated using the relative intensities of each monomer determined from IM (highlighted in red). 